\title{
Férias do treino? Cuidado, 2 semanas de interrupção provocam alterações fisiológicas e psicobiológicas!
}

\author{
Training vacation? Caution, two weeks of interruption training cause \\ physiological and psychobiological alterations!
}

\author{
Geovana Silva Fogaça Leite ${ }^{1}$, Marco Tulio De Mello², Segio Tufik³ ${ }^{3}$ Hanna Karen Moreira \\ Antunes $^{1^{*}}$
}

ARTIGO ORIGINAL | ORIGINAL ARTICLE

\begin{abstract}
Para investigar o impacto de duas semanas de destreino na capacidade aeróbia e nas respostas psicobiológicas de corredores, 16 voluntários adultos foram recrutados. Após uma avaliação clínica, os voluntários foram submetidos a uma avaliação da composição corporal, coleta de sangue, teste ergoespirométrico máximo inicial e posteriormente outro em carga constante na intensidade do limiar ventilatório 1 (LV-1), e responderam questionários que avaliaram a ansiedade, perfil de humor e dependência de exercício. Uma semana após o término dessas avaliações, os voluntários foram submetidos a um período de destreino sustentado por duas semanas, e ao término, foram avaliados utilizando o mesmo procedimento inicial. Os resultados revelaram que o destreino causou diminuição no consumo de oxigênio e na ventilação em 9.1 e $8.6 \%$ respectivamente, além disso, o destreino aumentou os sintomas negativos de humor como ansiedade, tensão, depressão e diminuiu o vigor. Após a realização do exercício, houve alívio dos sintomas negativos de humor e aumento do vigor. Conclui-se que a interrupção do treino por um período curto de duas semanas provoca diminuição na capacidade aeróbia e alterações psicobiológicas.

Palavras-chave: Humor; Atividade Física; Destreino.
\end{abstract}

\begin{abstract}
To investigate the impact of two weeks of detraining in aerobic capacity and the psychobiological responses of runners, 16 adult volunteers were recruited. After clinical assessment, the volunteers were submitted to body composition evaluate, blood collection, maximum cardiopulmonary exercise test and constant load test in ventilatory threshold intensity 1 (VT-1), and answered psychobiological questionnaires to evaluate anxiety, mood profile and exercise dependence. A week after these tests, the volunteers were submitted to a detraining period sustained by two weeks. At the end of this period, the volunteers answers the psychobiological battery again and the cardiopulmonary exercise test on VT-I intensity was repeated. Psychobiological tests were applied again after the completion of the exercise. The results revealed that the detraining period caused a decrease in oxygen consumption and ventilation at 9.1 and $8.6 \%$, respectively, furthermore, the detraining increased negative mood symptoms as anxiety, stress, depression, angerhostility and decreased vigor. After the exercise, a relief of negative mood symptoms and increased of vigor was observed. We conclude that the interruption of training for a short period of two weeks causes a decrease in aerobic capacity and promote psychobiological alterations.
\end{abstract}

Keywords: Mood; Physical Activity; Detraining.

Artigo recebido a 07.02.2015; Aceite a 27.08.2015

${ }^{1}$ Departamento de Biociências, Universidade Federal de São Paulo, UNIFESP, Campus Baixada Santista, Santos, SP, Brasil

${ }^{2}$ Escola de Educação Física, Fisioterapia e Terapia Ocupacional, Universidade Federal de Minas Gerais, UFMG, Belo Horizonte, MG, Brasil

${ }^{3}$ Departamento de Psicobiologia, Universidade Federal de São Paulo- UNIFESP, Campus São Paulo, São Paulo, $S P$, Brasil

* Autor correspondente: Rua Silva Jardim, 136 Vila Mathias, Santos - SP, Brasil. Código Postal: 11015-020. Email: hanna.karen@unifesp.br 


\section{INTRODUÇÃO}

A prática regular do exercício físico promove diversas adaptações benéficas no organismo humano que podem ser percebidas nos sistemas cardiorrespiratório, endócrino, muscular, ósseo e nervoso (Booher \& Smith, 2003; Rivera-Brown \& Frontera, 2012), permitindo que esses sistemas trabalhem de forma mais eficiente, tendo como resultado uma melhoria no estado de saúde do praticante (Warburton, Nicol, \& Bredin, 2006). No entanto, quando o treino físico é interrompido, muitas das adaptações obtidas são revertidas, estabelecendo-se um processo conhecido por destreino (Mujika \& Padilla, 2000a).

O destreino pode ocorrer por diferentes motivos, entre eles, período de férias do praticante, pausa em função de algum tipo de lesão, aposentadoria desportiva, como estratégia de recuperação adotado pelo treinador, similar a um descanso profilático, mas diferente do período de Taper que representa um período de redução do volume de treino e aumento e/ou manutenção da intensidade do treino em períodos que antecedem uma competição importantes (Mujika \& Padilla, 2003), entre outros.

As consequências do destreino podem variar de acordo com o tempo de interrupção do treino, sendo temporalmente estudadas com base nas modificações causadas por um curto período de destreino ( $<4$ semanas) e aquelas causadas por um longo período ( $>4$ semanas) (Mujika \& Padilla, 2000a; Mujika \& Padilla, 2000b).

Geralmente, as modificações no contexto fisiológico observadas durante o período de destreino ganham destaque nas pesquisas científicas, uma vez que compreender o impacto da interrupção do treino permite estabelecer estratégias de retomada da atividade, ponto este particularmente fundamental para atletas. Por outro lado, a interrupção do treino físico pode gerar um quadro de complexos sintomas psicobiológicos (Antunes, Terrão, \& De Mello, 2010), sendo estes agravados a medida que o tempo de interrupção avança. Entre os sintomas mais conhecidos, podemos citar aumentos de ansiedade, agitação, tensão, depressão, irritabilidade, raiva, fadiga, confusão mental, distresse psicológico e queda do vigor (Aidman \& Woollard, 2003; Berlin, Kop, \& Deuster, 2006; Chan \& Grossman, 1988; Glass et al., 2004; Kop, Weinstein, Deuster, Wittaker, \& Tracy, 2008; Morris, Steinberg, Sykes, \& Salmon, 1990; Poole, Hamer, Wawrzyniak, \& Steptoe, 2011).

Embora as alterações fisiológicas envolvidas com o destreino estejam bem documentadas, as alterações psicobiológicas associadas a interrupção do treino tem sido negligenciadas, e trabalhos associando as duas esferas (física e psicobiológica) são escassos, o que justifica a realização de estudos que analisem esses tópicos. É válido mencionar que a presença desses sintomas pode se tornar mais preocupante se o praticante for um dependente de exercício físico, pois notoriamente a interrupção do exercício físico pode caracterizar um período de abstinência da atividade (Antunes et al., 2010).

Neste sentido, considerando a gama de sintomas envolvidos com a interrupção do treino físico e a pouca atenção dada a eles, além das alterações fisiológicas relacionadas, o objetivo do presente estudo foi o de investigar o impacto de duas semanas de interrupção do treino físico na capacidade aeróbia e nas respostas psicobiológicas de corredores. Neste trabalho foi testada a hipótese de que um período curto de destreino já seria capaz de produzir prejuízos no perfil de humor em praticantes regulares de corrida.

\section{MÉTODO}

Antes de participar, todos os voluntários foram informados sobre os procedimentos, desconfortos e riscos, e posteriormente eles assinaram o Termo de Consentimento Livre e Esclarecido concordando com os procedimentos envolvidos no estudo. O estudo foi aprovado pelo Comitê de Ética em Pesquisa da Universidade Federal de São Paulo / Hospital São Paulo (\#01249/09).

\section{Amostra}

Dezesseis atletas de corrida, saudáveis, do sexo masculino e com experiência em provas longas (10k, 21k e Maratona) foram selecionados para este estudo baseado nos seguintes critérios: a) Sexo masculino; b) Idade entre 18-40 anos; c) 
Ser fisicamente ativo há pelo menos 2 anos com regularidade mínima de 2 horas de exercício, $5 \mathrm{x} /$ semana; d) Não ter histórico de doença ou desordens psiquiátricas; e) Não ter lesão nos últimos 6 meses; f) Não ser tabagista; g) Não apresentar alterações no Eletrocardiograma de repouso e no Teste Ergométrico.

\section{Procedimentos}

Antes de iniciar os procedimentos experimentais, em uma primeira visita, todos os voluntários foram submetidos a uma avaliação clínica conduzida pelo médico do laboratório, incluindo um Eletrocardiograma de repouso e de esforço. Os voluntários considerados aptos foram incluídos no estudo e assinaram o termo de consentimento livre e esclarecido.

$\mathrm{Na}$ segunda visita, os voluntários foram submetidos a uma avaliação da composição corporal, e na terceira visita, uma coleta de sangue e aplicação de questionários psicobiológicos foram realizados. $\mathrm{Na}$ quarta e quinta visita, foram realizados um teste ergoespirométrico máximo e um teste de carga constante por 60 minutos, respectivamente. As visitas foram separadas uma das outras por um intervalo mínimo de 48 horas. Essas análises constituíram o período Basal.

Uma semana após o término do período Basal, os voluntários iniciaram o período de destreino, que foi sustentado por 2 semanas consecutivas. Ao fim deste período, os voluntários responderam os questionários psicobiológicos, e posteriormente foram submetidos a um novo teste ergoespirométrico em carga constante por 60 minutos e novamente responderam os questionários psicobiológicos.

Para controle do destreino, ao fim da primeira semana (7 dias) e ao término das 2 semanas propostas pelo estudo, os voluntários foram submetidos a coleta de sangue. Durante todo o período de realização do protocolo, os voluntários usaram um actígrafo para monitorar o índice de movimento.

\section{Testes Físicos}

Um teste ergoespirométrico utilizando cargas progressivas até a exaustão voluntária máxima em esteira ergométrica (Lifefitness ${ }^{\circledR}$ 9700HR,
Schiller Park, IL, USA) para determinar a capacidade aeróbia dos voluntários foi realizado. Este teste foi realizado para determinar a capacidade aeróbia representada pelo consumo de oxigênio pico e a intensidade que corresponde ao Limiar Ventilátorio 1 (LV-1) que compreende a fase predominantemente aeróbica do esforço e consistiu em aumentos de velocidade de $1 \mathrm{~km} / \mathrm{h}$ a cada minuto, com aquecimento inicial de $7 \mathrm{~km} / \mathrm{h}$ por 3 minutos. O teste foi finalizado quando o voluntário alcançou a exaustão voluntária máxima.

Durante todo o teste foi utilizado $1 \%$ de inclinação para similar o desgaste físico em locais abertos (Jones \& Doust, 1996). Os parâmetros ventilatórios foram determinados utilizando um sistema metabólico (Quark PFT Pulmonary Function Testing, FRC and DLCO - 4Ergo Italy Cosmed $^{\circledR}$ ).

Os critérios descritos por Wasserman e Koike (1992) foram usados para determinar o LV-1. Durante todos os testes a frequência cardíaca (Polar $^{\circledR}$, model Advantage NV, Kempele, Finland) e o comportamento da pressão arterial (Duo-sonic estetoscópio, $\quad \mathrm{BD}^{\circledR}$ esfigmomanômetro), foram monitorados. Os testes foram realizados no mesmo período do dia (Carrier \& Monk, 2000), e foram conduzidos em laboratório com temperatura controlada (20$22^{\circ} \mathrm{C}$ ). Se o último estágio do teste não pudesse ser completado, a velocidade máxima foi calculada de acordo com a equação proposta por Kuipers, Rietjens, Verstaooen, Schoenmakerse, e Hofman, (2003). Após um intervalo mínimo de 48 horas, um segundo teste foi realizado, desta vez, para avaliar a capacidade aeróbio em exercício moderado, para isso, teste em carga constante por 60 minutos na intensidade do LV1 foi realizado. Este teste foi reaplicado novamente a pós o termino do período de destreino.

\section{Composição Corporal}

A composição corporal foi determinada utilizando o método de Bioimpedância (InBody720 ${ }^{\circledR}$ Biospace). Todos os procedimentos exigidos para a realização desta avaliação foram observados. 


\section{Análises Bioquímicas}

As coletas sanguíneas foram realizadas por meio de punção periférica de veia do antebraço por profissional especializado. As amostras foram coletadas em tubos de vidro contento EDTA como anticoagulante (Vacutainer $\left.{ }^{\circledR}, \mathrm{BD}\right)$, e centrifugadas para separar soro e plasma. As amostras foram estocadas em biofreezer a $-80^{\circ} \mathrm{C}$ até a realização das análises. As coletas foram realizadas nos momentos: basal, 7 dias e ao término do período de destreino. As variáveis analisadas foram: Creatina Fosfato (CPK) e Lactato Desidrogenase (LDH), medidos por imunoinibição (IFCC - Modified Szasz, Advia $2400^{\circledR}$ Centaur-Siemens $^{\circledR}$ ); e Proteína C-Reativa, medida por Nefelometria de alta sensibilidade (Image 800 ${ }^{\circledR}$ Beckman Coulter ${ }^{\circledR}$ ).

\section{Instrumentos}

\section{Visual Analogue Mood Scale}

Escala visual Analógica de humor, que avalia o perfil de humor de forma subjetiva. É composta por 16 escalas analógicas de $10 \mathrm{~cm}$ que liga dois adjetivos opostos, através das quais o avaliado por meio de um traço vertical avalia o melhor ponto que descreve seus sentimentos (Zuardi \& Karniol, 1981). As questões são agrupadas de forma a permitir a identificação de 4 fatores: ansiedade, sedação física, sedação mental, outros sentimentos e atitudes.

\section{Inventário Beck de Depressão}

Instrumento que avalia o estado de depressão (Gorenstein \& Andrade, 1996). É composto por 21 itens, os quais avaliam sintomas e atitudes que variam numa escala de pontuação de $0-3$. A classificação dos escores indica os seguintes escores indicativos para a depressão: normal (09), leve (10-15), leve a moderado (16-19), moderada a severa (20-29), severa (30-63).

\section{Inventário de Ansiedade Traço-Estado (IDATE)}

A ansiedade foi quantificada através de um Inventário de Ansiedade Traço-Estado (State Trait Anxiety Inventory - STAI) - Este instrumento auto avaliativo está dividido em duas partes: uma avalia a ansiedade-traço (referindo-se a aspectos de personalidade) e a segunda avalia a ansiedade-estado (referindo-se a aspectos sistêmicos do contexto). Cada uma dessas partes é composta de 20 afirmações. Ao responder o questionário, o indivíduo deve levar em consideração uma escala de quatro itens que variam de 1 a 4, sendo que ESTADO significa como o sujeito se sente no "momento" e TRAÇO como ele "geralmente se sente". O escore de cada parte varia de 20 a 80 pontos, sendo que os escores podem indicar um baixo grau de ansiedade (0-30), um grau mediano de ansiedade (31-49) e um grau elevado de ansiedade (maior ou igual a 50), quanto mais baixo se apresentarem os escores, menor será o grau de ansiedade (Biaggio \& Natalicio, 1979). É importante mencionar que nesse estudo, a parte referente à ansiedade Traço foi aplicada em um único momento (início do estudo), por referir ao traço de personalidade do voluntário.

\section{Escala de Dependência de Exercício (EDS-21)}

Desenvolvido por Hausenblas e Downs (2002) e validade para a população brasileira por Vasconcelos de Oliveira (2010), a Escala de Dependência de exercício (EDS-21), conceitua a prática do exercício compulsivo, baseado nos critérios do DSM-IV, para abuso e dependência de substâncias. $\mathrm{O}$ questionário permite diagnosticar se os participantes apresentam risco para dependência de exercício, se são nãodependentes sintomáticos ou não-dependentes assintomáticos. Os participantes indicam suas respostas em cada um dos 21 itens do questionário, numa escala tipo Likert sendo (1 nunca) e (5 - sempre), a partir da pontuação atingida nas 7 subescalas: tolerância/resistência, evitar sintomas de abstinência, intencionalidade, falta de controle, tempo, redução de outras atividades, continuidade, é dada a classificação. Com relação a cada subescala, pontuação igual a 15 indica dependência, 7 - 14 não dependente sintomático e abaixo de 7 assintomático. Para classificação de dependência de exercício, o sujeito precisa ser sintomático em pelo menos 3 subescalas. Além disso, o questionário aponta uma classificação quanto ao tipo de dependência sendo esta fisiológica quando há evidência nas subescalas "tolerância/resistência" e "evitar sintomas de abstinência”, e não fisiológica, quando não há evidencias nestas subescalas. 


\section{Análise Estatística}

Foi aplicado o teste Shapiro Wilk para verificação da normalidade dos dados e Teste de Levene para verificar a homogenidade das variâncias. Os resultados estão expressos em média \pm desvio-padrão ou porcentagem quando necessário. Os dados foram comparados através de Teste- $t$ para amostras dependentes (pareado), assim como com análise de variância ANOVA com o teste post-hoc Tukey quando necessário. Para todas as análises, o nível de significância adotado foi de $\mathrm{p} \leq 0.05$ e o tamanho do efeito para F está representado como partial eta-squared $\left(\eta^{2}\right)$, e para t foi calculado o Cohen's d. As análises foram realizadas com auxílio do software STATISTICA 12.0 (StatSoft, Inc., Tulsa, OK, USA).

\section{RESULTADOS}

Os voluntários apresentaram $\mathrm{VO}_{2 \text { pico, }}$ frequência cardíaca máxima, velocidade pico e IMC compatíveis com amostra de sujeitos jovens, saudáveis, ativos, eutróficos e com boa capacidade aeróbia. O Idate Traço apresentou classificação mediana para ansiedade.

Tabela 1

Características da Amostra

\begin{tabular}{|c|c|}
\hline Parâmetros & $\begin{array}{l}\text { Média } \pm \text { desvio } \\
\text { padrão }\end{array}$ \\
\hline Idade (anos) & $31.88 \pm 4.27$ \\
\hline Estatura (m) & $1.75 \pm 0.08$ \\
\hline Massa Corporal Total (kg) & $70.57 \pm 10.85$ \\
\hline Massa Magra (\%) & $86.48 \pm 5.49$ \\
\hline Massa Gorda (\%) & $13.52 \pm 5.49$ \\
\hline $\operatorname{IMC}\left(\mathrm{Kg} / \mathrm{m}^{2}\right)$ & $23.18 \pm 2.63$ \\
\hline Idate Traço & $32.88 \pm 4.77$ \\
\hline$\dot{\mathrm{V}} \mathrm{O}_{2 \text { pico }}\left(\mathrm{ml} \cdot \mathrm{kg} \cdot \mathrm{min}^{-1}\right)$ & $57.00 \pm 6.30$ \\
\hline FC máx (bpm) & $186.00 \pm 7.49$ \\
\hline Velocidade pico $(\mathrm{Km} / \mathrm{h})$ & $18.31 \pm 0.95$ \\
\hline$\dot{\mathrm{V}} \mathrm{O}_{2} \mathrm{LV}-1\left(\mathrm{~mL} \cdot \mathrm{kg} \cdot \mathrm{min}^{-1}\right)$ & $40.97 \pm 4.60$ \\
\hline Velocidade LV-1 (km/h) & $11.75 \pm 1.06$ \\
\hline$\dot{\mathrm{VO}}{ }_{2} \mathrm{LV}-2\left(\mathrm{~mL} \cdot \mathrm{kg} \cdot \mathrm{min}^{-1}\right)$ & $49.04 \pm 7.36$ \\
\hline Velocidade LV-2 (km/h) & $15.69 \pm 1.40$ \\
\hline $\begin{array}{l}\text { nálise descritiva, dados apr } \\
\text { adrão, referentes a } 16 \text { volunt } \\
\text { assa corporal; FC- Frequê } \\
\text { entilatório } 1 \text {; LV-2- Limiar Ve }\end{array}$ & $\begin{array}{l}\text { em média } \pm \text { desvi } \\
\text { da: IMC- índice } \\
\text { ca; LV-1- Limi } \\
\text {; máx- máxima. }\end{array}$ \\
\hline
\end{tabular}

Tabela 2

Análises Sanguíneas

\begin{tabular}{lccc}
\hline \multicolumn{1}{c}{ Parâmetros } & Basal & $7^{\circ}$ dia & $14^{\circ}$ dia \\
\hline Lactato Desidrogenase, U/L & $170.19 \pm 41.84$ & $152.00 \pm 32.50^{\mathrm{a}}$ & $147.75 \pm 29.97^{\mathrm{a}}$ \\
Creatina Fosfato, U/L & $255.81 \pm 171.60$ & $124.71 \pm 43.58^{\mathrm{a}}$ & $135.25 \pm 69.89^{\mathrm{a}}$ \\
Proteína C Reativa, mmol/L & $0.10 \pm 0.07$ & $0.08 \pm 0.10$ & $0.08 \pm 0.07$
\end{tabular}

Anova para medidas repetidas com Duncan como post hoc, $\mathrm{p}<0,05 .{ }^{a}-$ diferente do Basal. Dados apresentados em média \pm desvio-padrão, dados referentes a 16 voluntários.

Na tabela 2, são apresentados a média das concentrações das enzimas LDH, CPK e Proteica C Reativa. Para a enzima LDH, observamos diferenças em relação ao tempo $\left(F_{(2,24)}=3.99\right.$; $\left.\mathrm{p}=0.03, \quad \eta^{2}=0.24\right)$, onde as concentrações observadas no $14^{\circ}$ dia foram menores do que aquelas encontradas no momento basal $(\mathrm{p}=0,001)$. Para a CPK $\left(F_{(2,24)}=6.86 ; \mathrm{p}=0.01\right.$, $\left.\eta^{2}=0.36\right)$, as diferenças em relação ao tempo revelaram menores concentrações em relação basal, nos momento $7^{\circ}$ dia e $14^{\circ}$ dia $(\mathrm{p}=0,01$, ambos). As outras análises não apresentaram diferenças significativas.
Os dados referentes a capacidade aeróbia quando comparados o teste em carga constante na intensidade do LV-1 realizado no momento basal e logo após o término do período de destreino, estão apresentados na figura 1. A comparação revelou que o período de destreino causou uma diminuição de $9.1 \%$ no consumo de oxigênio relativo $(t=2.89 ; \mathrm{p}=0.01$, Cohen's $\mathrm{d}=$ $0.99)$ e de $8.6 \%$ na ventilação $(t=2.15 ; p=0.04$, Cohen's $d=0.66)$. As outras medidas não apresentaram mudanças significativas. 

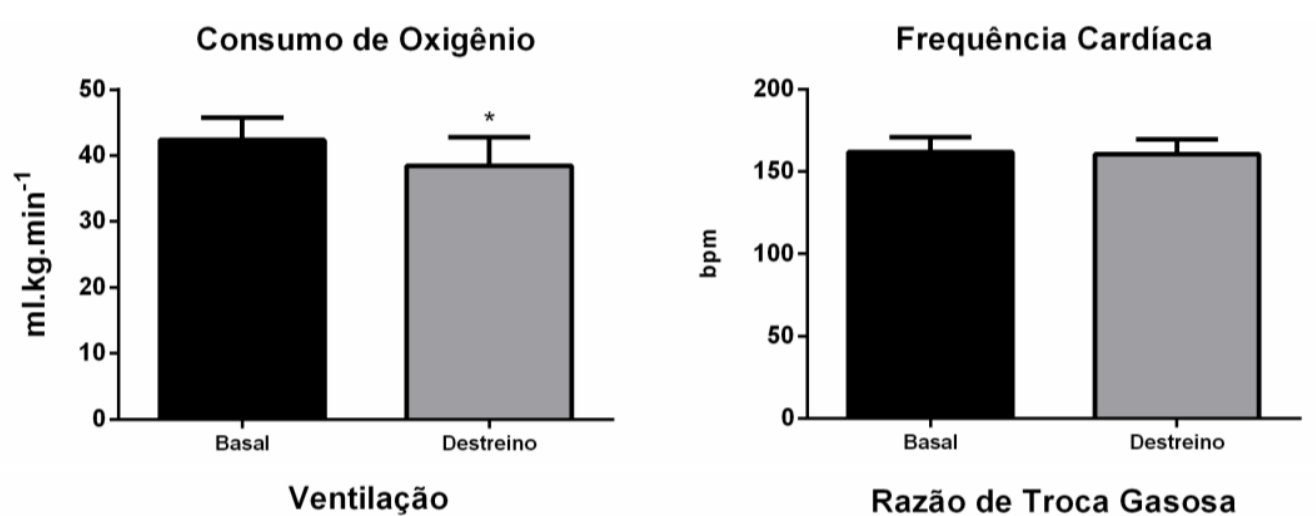

Razão de Troca Gasosa
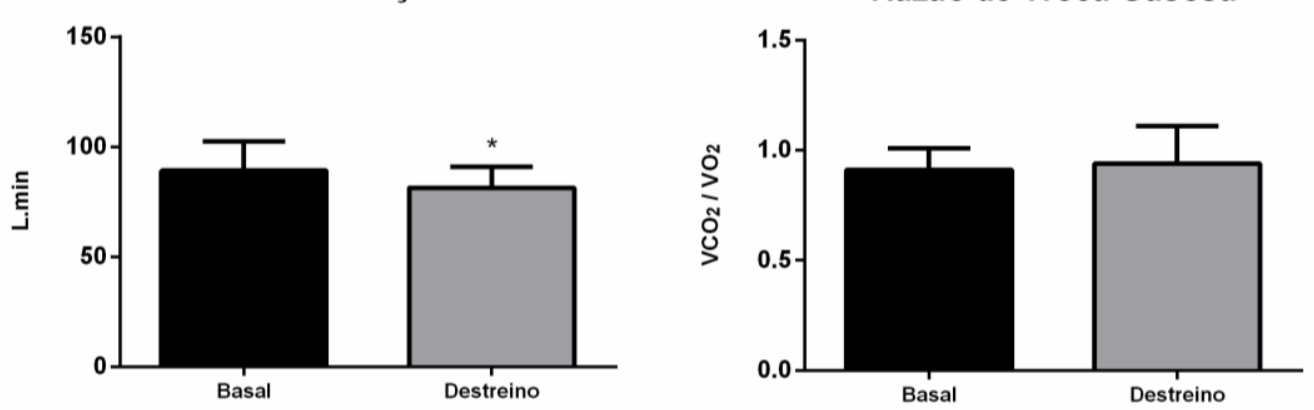

Figura 1. Capacidade Aeróbia em Carga Constante. Teste- $t$ para amostras dependentes. * - diferente do Basal. Resultados significativos para $\mathrm{p} \leq 0.05$. Dados apresentados em média \pm desvio-padrão. Dados referentes a 16 voluntários.

Tabela 3

Questionário de Dependência de Exercício Físico

\begin{tabular}{|c|c|c|}
\hline \multirow{8}{*}{$\begin{array}{l}\overrightarrow{7} \\
\stackrel{2}{2} \\
\stackrel{1}{2}\end{array}$} & Tolerância/Resistência & $10.88 \pm 2.22$ \\
\hline & Evitar sintomas de abstinência & $9.19 \pm 3.02$ \\
\hline & Intencionalidade & $7.38 \pm 2.28$ \\
\hline & Falta de Controlo & $10.25 \pm 2.44$ \\
\hline & Tempo & $8.31 \pm 2.77$ \\
\hline & Redução de outras Atividades & $7.13 \pm 3.12$ \\
\hline & Continuidade & $6.13 \pm 2.39$ \\
\hline & Escore Global & $59.25 \pm 13.32$ \\
\hline
\end{tabular}

Análise descritiva, dados apresentados em média \pm desviopadrão, referentes a 16 voluntários.

A tabela 3 apresenta os resultados dos escores observados nas subescalas do Questionário de Dependência de Exercício (EDS-21). Em relação aos escores das subescalas, os resultados revelaram que nossa amostra era composta por indivíduos não-dependentes, porém sintomáticos. Os critérios de análise da escala permitiram identificar que $37.5 \%$ da amostra podem ser considerados dependentes de exercício, enquanto que $56.25 \%$ e $6.25 \%$ são nãodependentes sintomáticos e assintomáticos respectivamente. Além disso, quanto à evidência de sintomas de tolerância e retirada, e $68.75 \%$ da amostra apresentaram esses sintomas, sendo caracterizadas como sintomáticos para dependência fisiológica, enquanto que $31.25 \%$ não apresentaram evidencias destes sintomas.

Tabela 4

Variáveis Psicobiológicas

\begin{tabular}{|c|c|c|c|c|}
\hline \multicolumn{2}{|c|}{ Variáveis } & Basal & Destreino & Após o Exercício \\
\hline \multicolumn{2}{|c|}{ Idate Estado } & $31.94 \pm 6.81$ & $43.46 \pm 11.54^{\mathrm{a}}$ & $31.64 \pm 5.65^{\mathrm{b}}$ \\
\hline \multirow{6}{*}{ 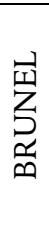 } & Tensão & $2.13 \pm 1.63$ & $6.79 \pm 8.55^{\mathrm{a}}$ & $1.43 \pm 2.06^{\mathrm{b}}$ \\
\hline & Depressão & $0.44 \pm 0.89$ & $3.14 \pm 4.40^{\mathrm{a}}$ & $0.07 \pm 0,27^{b}$ \\
\hline & Raiva- Hostilidade & $0.63 \pm 1.26$ & $3.57 \pm 4.16^{\mathrm{a}}$ & $0.93 \pm 2.23^{b}$ \\
\hline & Vigor & $11.31 \pm 1.99$ & $8.36 \pm 3.25^{\mathrm{a}}$ & $11.29 \pm 2.33^{\mathrm{b}}$ \\
\hline & Fadiga & $2.56 \pm 2.28$ & $3.79 \pm 3.89$ & $2.36 \pm 3.18$ \\
\hline & Confusão Mental & $0.81 \pm 1.33$ & $2.21 \pm 2.52$ & $1.00 \pm 2.00$ \\
\hline \multirow{4}{*}{$\sum_{>}^{\infty}$} & Ansiedade & $61.87 \pm 1.04$ & $56.89 \pm 15.60$ & $62.12 \pm 1.11$ \\
\hline & Sedação Física & $56.24 \pm 1.56$ & $55.99 \pm 2.25$ & $56.18 \pm 0.61$ \\
\hline & Sedação Mental & $54.45 \pm 0.99$ & $54.54 \pm 1.07$ & $54.87 \pm 0.71$ \\
\hline & Outros Sentimentos & $53.99 \pm 0.82$ & $53.30 \pm 1.23$ & $54.04 \pm 0.68$ \\
\hline
\end{tabular}

Anova para medidas repetidas com Duncan como post hoc, $\mathrm{p}<0,05 .{ }^{\mathrm{a}}$ - diferente do Basal; ${ }^{\mathrm{b}}-$ diferente do Destreino. Dados apresentados em média \pm desvio-padrão. 
$\mathrm{Na}$ tabela 4, apresentamos os resultados das variáveis psicobiológicas. Em relação ao Idate Estado, observamos diferenças em relação ao tempo $\left(F_{(2,20)}=18.20 ; \mathrm{p}=0.01, \eta^{2}=0.64\right)$, onde os escores de ansiedade aumentaram em função do período de destreino $(\mathrm{p}=0.01)$, retornando aos valores encontrados no basal imediatamente após o exercício $(\mathrm{p}=0.01)$. A análise do perfil de humor realizado pelo Brunel, revelou que o destreino provocou aumentos nos escores das dimensões Tensão $\quad\left(F_{(2,22)}=3.84 ; \mathrm{p}=0.03\right.$, $\left.\eta^{2}=0.25\right)$, quando comparado com basal $(\mathrm{p}=0.04)$, diminuindo logo após a realização do exercício físico $(\mathrm{p}=0.02)$. Para a dimensão Depressão $\left(F_{(2,22)}=3.65 ; \mathrm{p}=0.04, \quad \eta^{2}=0.24\right)$ e Raiva-Hostilidade $\quad\left(F_{(2,22)}=4.88 ; \quad \mathrm{p}=0.01\right.$, $\eta^{2}=0.30$ ), comportamento similar foi observado, com aumentos causados pelo destreino $(\mathrm{p}=0.03$ e $\mathrm{p}=0.01$ respectivamente) seguido de diminuição após o exercícios $(\mathrm{p}=0.02$ e $\mathrm{p}=0.01$ respectivamente).

Além disso, observamos que a dimensão Vigor $\quad\left(F_{(2,22)}=6.37 ; \quad \mathrm{p}=0.01, \quad \eta^{2}=0.36\right)$ apresentou uma diminuição com o destreino, retornando aos valores basais após a realização do exercício físico ( $\mathrm{p}=0.01$ ambos). As outras análises não revelaram alterações significativas.

\section{DISCUSSÃO}

Os resultados observados neste estudo demonstraram que um período curto de destreino (2 semanas) foi capaz de prejudicar a capacidade aeróbia de corredores e ao mesmo tempo aumentar os sintomas negativos de humor, sendo este último amenizado após realização do exercício físico, indicando um efeito imediato do exercício no alívio destes sintomas. É válido mencionar que a amostra utilizada neste estudo foi composta por corredores com boa capacidade aeróbia, eutróficos e muito engajados com esta atividade, no entanto não classificados como dependentes de exercício físicos.

Para testar a adesão ao protocolo de destreino e garantir que os voluntários não se exercitaram durante o período do estudo, foram realizadas avaliações das enzimas LDH e CPK que são marcadores que representam medidas da condição funcional do tecido muscular e do dano celular respectivamente (Brancaccio, Maffulli, Buonauo, \& Limongelli, 2008), e da Proteína C Reativa que é uma proteína secretada pelo fígado e um indicador sensível de inflamação. Em situação de exercício, isto é, quando a prática é regular, esses marcadores se mantem aumentados em relação a uma condição onde esta prática não ocorre (Silva \& Macedo, 2011). As concentrações encontradas no $7^{\circ}$ e $14^{\circ}$ dias de destreino tanto para CPK quanto para a $\mathrm{LDH}$ foram menores quando comparado ao basal, no entanto, as concentrações da Proteina C-Reativa embora menores, não foram diferentes do ponto de vista estatístico. Esses resultados garantem que os voluntários não se exercitaram durante o protocolo experimental, assim podemos assegurar o período de interrupção do treino foi respeitado.

Como esperado, o destreino causou redução da capacidade aeróbia retratada por uma diminuição no consumo de oxigênio $(9.1 \%)$ e na ventilação (8.6\%) durante o exercício contínuo. Esses resultados estão de acordo com os descritos por Mujika e Padilla (2000a) que em sua revisão relatam que reduções no consumo de oxigênio entre $4-14 \%$ são comuns mesmo em períodos curtos em que o exercício físico não é praticado. Entre os parâmetros que podem auxiliar a justificar essas mudanças podemos sugerir uma redução do volume sanguíneo com redução do conteúdo proteico, associado a uma redução do volume sistólico e débito cardíaco, que tem como produto final um impacto negativo no desempenho aeróbio (Mujika \& Padilla, 2001).

Em relação aos aspectos psicobiológicos, o que chama atenção nos resultados foi a similaridade entre os resultados do Idate Estado (ansiedade) e da Escala de Humor de Brunel (perfil de humor). Nestes instrumentos, tanto a ansiedade quanto o perfil de humor tiveram comportamentos similares, com aumentos dos sintomas negativos de humor e queda do vigor no período de destreino seguido de alívio desses sintomas imediatamente após a realização do exercício físico. Esses resultados reforçam a ocorrência de alterações no estado de humor causados pela interrupção do exercício (Antunes 
et al., 2010; Berlin et al., 2006; Kop et al., 2008; Poole et al., 2011), e o papel deste no alívio desses sintomas que provavelmente ocorrem em função de uma diminuição do estado de tensão comumente causado pela interrupção do exercício (Szabo, 1998). Devido a grande implicação prática, estes resultados representam um alerta aos profissionais da Educação Física e aqueles envolvidos com o exercício físico, uma vez que interrupções curtas no programa de treino ocorrem de forma corriqueira. Neste sentido, monitorizar as respostas psicobiológicas dos praticantes de exercício físico, bem como a presença de sintomas de dependência de exercício físico podem contribuir com este profissional no sentido de estabelecer uma estratégia para minimizar os prejuízos aqui descritos. Como limitação do estudo apontamos o número pequeno de voluntários analisados, e a ausência de um grupo controle nas comparações realizadas, por outro lado, destacamos a dificuldade de realização deste tipo de procedimento experimental que envolve a interrupção do período de treino em função da recusa de atletas. Além disso, consideramos também o fato dos voluntários conhecerem os objetivos do estudo, por se tratar de medida de variáveis psicobiológicas, é possível que isto tenha influenciado de alguma forma nos resultados encontrados.

\section{CONCLUSÕES}

Como conclusão, este trabalho demonstrou que a interrupção do treino por um período curto de duas semanas impacta negativamente na capacidade aeróbia reduzindo o consumo de oxigênio e produz aumentos no humor negativo (ansiedade, tensão, depressão, raiva-hostilidade) e diminuição do vigor.

\section{Agradecimentos:}

Os autores agradecem o apoio científico de Everald Vancouler e o apoio técnico da AFIP e do CEPE.

\section{Conflito de Interesses:}

Nada a declarar.

\section{Financiamento:}

FAPESP (11/04984-0; \#11/13592-8), CAPES, CNPq (\#475074/2011-4)

\section{REFERÊNCIAS}

Aidman, E., \& Woollard, S. (2003). The Influence of Self-reported Exercise Addiction on Acute Emotional and Physiological Responses to Brief Exercise Deprivation. Journal of Sport and Exercise, 4(3), 225-236. doi:10.1016/S14690292(02)00003-1.

Antunes, H.K.M., Terrão, F.L., \& De Mello, M.T. (2010). Efeitos e sintomas da privação do exercício físico - revisão. Revista Brasileira de Ciências Médicas e Saúde, 1(1), 53-61.

Berlin, A.A., Kop, W.J., \& Deuster, P.A. (2006). Depressive mood symptoms and fatigue after exercise withdrawal: the potential role of decreased fitness. Psychosomatic Medicine, 68(2), 224-30.

doi: 10.1097/01.psy.0000204628.73273.23.

Biaggio, A.M.B., \& Natalício, L. (1979). Manual para o Inventário de Ansiedade Traço Estado (IDATE). Rio de Janeiro: CEPA.

Booher, M.A., \& Smith, B.W. (2003). Physiological effects of exercise on the cardiopulmonary system. Clinics in Sports Medicine, 22(1), 1-21. doi:10.1016/S0278-5919(02)00034-0.

Brancaccio, P., Maffulli, N., Buonauro, R., \& Limongelli, F.M. (2008). Serum enzyme monitoring in sports medicine. Clinics in Sports Medicine, 27(1), 1-18. doi: 10.1016/j.csm.2007.09.005.

Carrier, J., \& Monk, T.H. (2000). Circadian rhythms of performance: new trends. Chronobiology International, 17(6), 719-32. doi: 10.1081/CBI100102108.

Chan, C.S., \& Grossman, H.Y. (1988). Psychological effects of running loss on consistent runners. Perceptual and Motor Skills, 66(3), 875-83. doi: 10.2466/pms.1988.66.3.875.

Glass, J.M., Lyden, A.K., Petzke, F., Steind, P., Whalene, G., Ambroseb, K., Chrousosf, G., \& Clauw, D.J. (2004). The effect of brief exercise cessation on pain, fatigue, and mood symptom development in healthy, fit individuals. Journal of Psychosomatic Research, 57(4), 391-398. doi:10.1016/j.jpsychores.2004.04.002.

Gorenstein, C., \& Andrade, L. (1996). Validation of a Portuguese version of the Beck Depression Inventory and State-Trait Anxiety Inventory in Brazilian Subjects. Brazilian Journal of Medical and Biological Research, 29(4), 453-457.

Hausenblas, H.A., \& Downs, D.S. (2002). How much is too much? The development and validation of the exercise dependence scale. Psychology \& Health, 17(4), 387-404. doi:10.1080/0887044022000004894. 
Jones, A.M., \& Doust, J.H. (1996). A 1\% treadmill grade most accurately reflects the energetic cost of outdoor running. Journal of Sports Science, 14(4), 321-327. doi:10.1080/02640419608727717.

Kop W.J., Weinstein, A.A., Deuster, P.A., Whittaker, K.S., \& Tracy R.P. (2008). Inflammatory markers and negative mood symptoms following exercise withdrawal. Brain, Behavior and Immunity, 22(8), 1190-1196. doi: 10.1016/j.bbi.2008.05.011.

Kuipers H., Rietjens, G., Verstappen, F., Schoenmakers, H., \& Hofman, G. (2003). Effects of stage duration in incremental running tests on physiological variables. International Journal Sports Medicine, 24(7), 486-491. doi: 10.1055/s2003-42020.

Morris, M., Steinberg, H., Sykes, E.A., \& Salmon, P. (1990). Effects of temporary withdrawal from regular running. Journal of Psychosomatic Research, 34(5), 493-500. doi: http://dx.doi.org/10.1016/0022-3999(90)90023W.

Mujika I., \& Padilla, S. (2000a). Detraining: loss of training-induced physiological and performance adaptations. Part I: short term insufficient training stimulus. Sports Medicine, 30(2), 79-87. doi: 10.2165/00007256-200030020-00002.

Mujika I., \& Padilla, S. (2000b). Detraining: loss of training-induced physiological and performance adaptations. Part II: long term insufficient training stimulus. Sports Medicine, 30(3), 145-154. doi: 10.2165/00007256-200030030-00001.

Mujika I., \& Padilla, S. (2003). Scientific Bases for Precompetition Tapering Strategies. Medicine and Science in Sports and Exercise, 35(7), 1182-1187. doi: 10.1249/01.MSS.0000074448.73931.11.

Mujika, I., \& Padilla, S. (2001). Cardiorespiratory and metabolic characteristics of detraining in humans.
Medicine and Science Sports Exercise, 33(3), 413421.

Poole, L., Hamer, M., Wawrzyniak, A.J., \& Steptoe, A. (2011). The effects of exercise withdrawal on mood and inflammatory cytokine responses in humans. Stress, 14(4), 439-447. doi: 10.3109/10253890.2011.557109.

Rivera-Brown, A.M., \& Frontera, W.R. (2012). Principles of exercise physiology: responses to acute exercise and long-term adaptations to training. $P M \& R, \quad 4(11), \quad 797-804$. doi: 10.1016/j.pmrj.2012.10.007.

Silva, F.O.C., \& Macedo, D.V. (2011). Exercício físico, processo inflamatório e adaptação: uma visão geral. Revista Brasileira de Cineantropometria e Desempenho Humano, 13(4), 320-328. doi: 10.5007/1980-0037.2011v13n4p320.

Szabo, A. (1998). Studying the psychological impact of exercise deprivation: Are experimental studies hopeless? Journal Sport Behavior, 21, 139-147.

Vasconcelos de Oliveira, I. C. (2010). Adaptação e Validação da Escala de Dependência de Exercício Físico em versão tradicional e informatizada (Dissertação de Mestrado). Universidade Federal do Rio Grande do Norte, Natal.

Warburton, D. E. R., Nicol, C. W., \& Bredin, S. S. D. (2006). Health benefits of physical activity: the evidence. Canadian Medical Association Journal, 174(6), 801-809. https://doi.org/10.1503/cmaj.051351

Wasserman, K., \& Koike, A. (1992). Is the anaerobic threshold truly anaerobic? Chest, 101(5 Suppl), 211S-218S.

Zuardi, A. W., \& Karniol, I. G. (1981). Estudo transcultural de uma escala de auto-avaliacao para estados subjetivos. Jornal Brasileiro de Psiquiatria, 30(5), 403-6. 